\title{
Biochemical Characterization of a Trypanosomatid Isolated from the Plant Amaranthus retroflexus
}

\section{Clotilde Marín, Carolina Fernández-Ramos, Emilio Entrala, José M Quesada, Manuel Sánchez-Moreno ${ }^{+}$}

Instituto de Biotecnología, Grupo de Bioquímica y Parasitología Molecular, Facultad de Ciencias, Universidad de Granada, E-18071 Granada, España

A protozoan flagelate has recently been isolated from Amaranthus retroflexus. This plant grows near economically important crops in southeastern Spain, which are known to be parasitized by Phytomonas spp. The present study focuses on the characterization of the energy metabolism of this new isolate. These flagellates utilize glucose efficiently as their primary energy source, although they are unable to completely degrade it. They excrete ethanol, acetate, glycine, and succinate in lower amount, as well as ammonium. The presence of glycosomes was indicated by the early enzymes of the glycolytic pathway, one enzyme of the glycerol pathway (glycerol kinase), and malate dehydrogenase. No evidence of a fully functional citric-acid cycle was found. In the absence of catalase activity, these flagellates showed significant superoxide dismutase activity located in the glycosomal and cytosolic fractions. These trypanosomes, despite being morphologically and metabolically similar to other Phytomonas isolated from the same area, showed significant differences, suggesting that they are phylogenetically different species.

Key words: Amaranthus retroflexus - Phytomonas - energy metabolism - superoxide dismutase

It is known that plants can be parasitized by members of the Trypanosomatidae family, artificially grouped into the genus Phytomonas by Donovan (1909) to differentiate plant from animal trypanosomatids. Our group has isolated plant flagellates from different economically important fruit crops in southeastern Spain (Sanchez-Moreno et al. 1995), and they were consequently classified as members of the genus Phytomonas (Uttaro et al. 1997). In another study, we demonstrated that flagellates from different fruits have a common origin and that the same flagellates parasitize several species of plants (Fernandez-Becerra et al. 1997). Now, we sampled wild plants near the crops to determine whether these could serve as reservoirs for the parasites. In this way, we isolated two new flagellates, one in the stems of clover (Trifolium glomeratum) and another in the stems of amaranth (Amaranthus retroflexus). Both isolates were characterized by isoenzymatic analysis and by kDNA analysis using five different restriction endonucleases, verifying these with the isolates from tomato, cherimoya and mango fruits in the same zone (Sanchez-Moreno et al. 1998).

\footnotetext{
${ }^{+}$Corresponding author. Fax: +34-9-58-243.174. E-mail: msanchem@goliat.ugr.es

Received 4 November 1999

Accepted 9 February 2000
}

The isolates from clover were identical to those isolated from the fruits, but the isolates from amaranth were completely different. These isolates presented activity of iso-propanol dehydrogenase, the marker enzyme of the genus Phytomonas (Uttaro et al. 1997). In the present work, we analyse these isolates from a metabolic standpoint to test whether they constitute a species different from that found in tomato, cherimoya and mango fruits and in clover stems.

\section{MATERIALS AND METHODS}

Organisms and in vitro culture - Vegetative samples from $A$. retroflexus were collected from the southern coast of Spain, in the hydrogenic crops. Small sections of the roots, stems and leaves were homogenized in phosphate buffered saline and examined by phase-contrast microscopy. The samples founds to be infected with flagellates (stems) were cultivated in $25 \mathrm{ml}$ of Grace's medium supplemented with $10 \%$ heat-inactivated foetal calf serum, as previously described Sanchez-Moreno et al. (1998). Aliquots of $5 \mathrm{ml}$ were taken every $24 \mathrm{~h}$ for four days during the experiment. The number of parasites was determined in a Neubauer haemocytometric chamber, after which the cells were removed by centrifugation at $600 \mathrm{x}$ g for $10 \mathrm{~min}$. The $\mathrm{pH}$ of the parasite-free supernatants was measured before they were frozen at $-80^{\circ} \mathrm{C}$ for subsequent measurements of glucose consumption, using the enzyme method of Bergmeyer (1988). 
${ }^{1} H$ NMR spectroscopy and metabolite identification - For the spectroscopic studies, $5 \mathrm{ml}$ of a four-day-old culture were centrifuged at $1,500 \mathrm{~g}$ for $10 \mathrm{~min}$ in at $4^{\circ} \mathrm{C}$. The pellet was discarded, and the supernatant, freed of parasites, was stored at $-20^{\circ} \mathrm{C}$ until use for ${ }^{1} \mathrm{H}$ NMR. The ${ }^{1} \mathrm{H}$ NMR spectra were obtained at $300 \mathrm{Mhz}$ on a Bruker AM-300 spectrometer, which was operated at pulse mode in Fourier transformation with quadrature detection (Gilroy et al. 1988). The temperature of the probe was maintained at $27^{\circ} \mathrm{C}$. The acquisition parameters were pulses of $90^{\circ}$ in radius and a wavelength of $3,287.5 \mathrm{~Hz}, 8 \mathrm{sec}$ recycle time and 160 accumulations. The chemical displacements were expressed as part per million (ppm) relative to a tetramethyl xylane signal.

The chemical displacements used to identify the respective metabolites were consistent with those described in the literature (Sánchez-Moreno et al. 1992). The resonance of the peaks observed in the spectra were assigned to a given metabolite by the addition of standards to the fresh medium and the measurement of their chemical shifts.

Metabolites were quantified enzymatically as described by Bergmeyer (1988). All the experiments and determinations were repeated five times.

Cell fractionation - Homogenates of trypanosomatids were prepared by grinding the cell paste with silicon carbide abrasive grain. After cell lysis, centrifugation was carried out following the method described by Steiger et al. (1980). The different subcellular fractions were obtained as follows: unbroken cells and nuclei were sedimented at $1,500 \mathrm{~g}$ for $10 \mathrm{~min}$ (nuclear fraction: $\mathrm{N}$ ). From the resulting cytoplasmatic extract (post-nuclear: $\mathrm{PN}$ ), a granular fraction was obtained by centrifugation at 5,000 $\mathrm{g}$ for $10 \mathrm{~min}$ (large organelles: $\mathrm{LG}$ ). The supernatant was centrifugated at $14,500 \mathrm{~g}$ for $10 \mathrm{~min}$, and the sediment kept as the smallorganellar fraction (SG), while the supernatant was again centrifugated at $140,000 \mathrm{~g}$ for $60 \mathrm{~min}$. The pellet was resuspended and kept as the microsomal fraction (M). The remaining supernatant was kept as the soluble fraction (S). For each of the different fractions obtained, the concentration of proteins was determined by the Bio-Rad test, based on the method of Bradford (1976) and aliquots were stored at $-80^{\circ} \mathrm{C}$ until used. All the centrifugations and manipulation of the samples were performed at $4^{\circ} \mathrm{C}$.

Enzyme determinations - hexokinase (HK), pyruvate kinase (PK), phosphofructokinase (PFK), phosphoglucose isomerase (PGI), glyceraldehydephosphate dehydrogenase (GADPH), triosephosphate isomerase (TIM), fructose 1,6-biphosphate aldolase (ALD) and the enzyme of glycerol metabolism, glycerol kinase (GK) were determined according to Misset et al. (1986). Pyruvate kinase
(PK) activity was measured after the addition of fructose 2,6-bisphosphate as described by Van Schaftingen et al. (1985). Malate dehydrogenase (MDH) was assayed according to Opperdoes et al. (1981). Fumarase (FUM) was measured according to Klein et al. (1975) and superoxide dismutase (SOD) activity was assayed by Paoleti and Moccali (1990).

Electrophoresis - SOD activity was also demonstrated using an on gel assay. The proteins were separated on $8-25 \%$ native polycrylamide gradient gels and on $\mathrm{pH} 3$ isoelectric focusing gels using a Pharmacia Phast-system. SOD activity staining was carried out with the same reagents as the NBT assay (Beyer \& Fridovich 1987). Gels were soaked in $10 \mathrm{ml}$ of the stock solution and illuminated with UV light until enzyme activity appeared as a colourless band on a blue background.

To distinguish between $\mathrm{Cu}-\mathrm{Zn}$ and $\mathrm{Mn}$ or $\mathrm{Fe}$ dependent SOD, activity was measured on gel assay in the presence of $20 \mathrm{mM}$ potassium cyanide and after treatment with $5 \mathrm{mM} \mathrm{H}_{2} \mathrm{O}_{2}, 1$ mM EDTA for $45 \mathrm{~min}$ at $37^{\circ} \mathrm{C}$. Molecular weight was visualized by silver staining (Pharmacia).

\section{RESULTS AND DISCUSSION}

Flagellates isolated from A. retroflexus and adapted to the in vitro culture presented a growth curve similar to that described for other flagellates isolated from tomato and cherimoya fruits (Sánchez-Moreno et al. 1995). They exhibited an exponential growth phase during the first four days of culture (Fig. 1A) followed by the stationary phase, reaching a cell density of $2.10^{7}$ cells $/ \mathrm{ml}$ by day 4 of the culture.

The flagellates included in the genus Phytomonas, like other trypanosomatids, depend primarily on glycolysis to obtain energy (SánchezMoreno et al. 1992). This capacity to utilize glucose is a characteristic that allows them optimal adaptation to the host, especially in view of the fact that these parasites lack a system for carbohydrate storage. In the flagellates isolated from amaranth, glucose consumption from their medium proved inversely proportional to their growth (Fig. 1B). Parallel to this consumption was a significant drop in the $\mathrm{pH}$ of the medium (Fig. 1B), reaching the strongest acidification at the beginning of the stationary phase. This was because the glucose was not completely degraded to $\mathrm{CO}_{2}$ and part of the carbon skeleton was excreted as fermentative metabolites, as demonstrated in other flagellates (Sánchez-Moreno et al. 1992, Blum 1993).

To identify these metabolites, we used ${ }^{1} \mathrm{H}$ NMR spectroscopy and, when the spectra of the cell-free media in which the parasite had grown for four days (Fig. 2B) were compared with those of fresh 


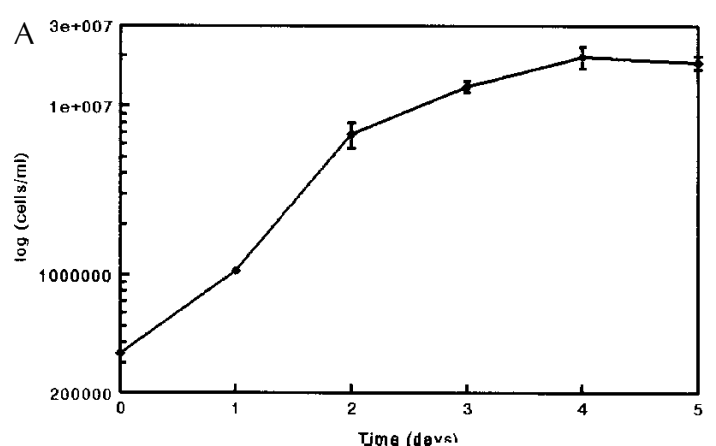

B

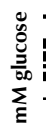

Time (days)

Fig. 1-A: growth curve of flagellates isolated from Amaranthus retroflexus, cultured in Grace's medium; B: glucose consumption (- $\boldsymbol{\Delta}$-), $\mathrm{pH}$ variation of Grace's medium during the growth of flagellates isolated from A. retroflexus (- - ) and excretion of $\mathrm{NH}_{4}^{+}(-\triangle-)$.

medium (Fig. 2A), we found a series of additional peaks which corresponded to metabolites excreted into the medium. Acetate (1.90 ppm), ethanol (1.20 ppm), glycine (3.63 ppm) and succinate (2.40 ppm) were the only metabolites excreted by the isolates from A. retroflexus. The production of ethanol, acetate and glycine (Fig. 3) was continuous over all four days of the culture, while the production of succinate increased during the first two days of the culture and remained constant thereafter. Acetate production as the main metabolite may reflect the inability to reoxidize this metabolite (Hellemond et al. 1998) as shown for other species of Phytomonas (Fernández-Becerra et al. 1996), although how acetate is produced remains unknown. Ethanol production could be the result either of a partial anaerobiosis in the cell culture during the growth phase or of the inability of the L-a-glycerolphosphate oxidase to eliminate all the reducing equivalents produced during glycolysis. The metabolic capacity of the Phytomonas species is quite limited, as the Krebs cycle is not active in the mitochondria (Sánchez-Moreno et al. 1992), and consequently Phytomonas cannot produce succinate by the inverse pathway of the Krebs cycle, as demonstrated in other trypanosomes (Fairlamb \& Opperdoes 1986). Therefore, succinate is produced as an end product because one part of the glyoxylic cycle is active in these organisms (Sánchez-Moreno et al. 1992). Another aspect of the intermediary metabolism of plant flagellates that has received some attention is ornithine-arginine metabolism. Trypanosomatid genera are differently equipped to promote the interconversion of ornithine-citruline-arginine, a metabolic cycle equivalent to the urea or Krebs cycle of vertebrates (Camargo 1999). Trypanosomatids depending on their enzymatic repertoir require one of these amino acids in their grouth medium to grow, and excrete either urea or ammonia, depending on their enzymatic repertory. Studies on the urea cycle enzymes of latex and fruit flagellates have disclosed that the enzymatic make-up of Phytomonas spp. is similar to that of Herpetomonas spp. in that they lack arginase and have the arginine deaminase-citrulline hydrolase system for converting arginine to ornithine, $\mathrm{CO}_{2}$ and ammonia. This system seems to operate also for the flagellates isolated from $A$. retroflexus, since these are capable of excreting ammonium into the culture medium (Fig. 1B).

The enzymatic study of the total homogenates of these flagellates revealed the presence of the principal enzymes of the glycolytic pathway (Table): HK, PK, PFK, GPI, GADPH, TIM, ALD, GK and MDH. However, FUM activity was not

\section{TABLE}

Specific activities of enzymes in homogenates of flagellates isolated from Amaranthus retroflexus

\begin{tabular}{lc}
\hline Enzyme & Specific activity $^{a}(\mathrm{mU} / \mathrm{mg})$ \\
\hline GK & 740.6 \\
HK & 684.0 \\
PK & 154.1 \\
PFK & 5299.8 \\
PGI & 922.2 \\
GAPDH & 2323.9 \\
ALD & 989.8 \\
TIM & 91.7 \\
MDH & 1910.6 \\
SOD & 40.1 \\
FUM & n.d. ${ }^{\text {b }}$
\end{tabular}

GK: glycerol kinase; HK: hexokinase; PK: pyruvate kinase; PFK: phosphofructokinase; PGI: phosphoglucose isomerase; GAPDH: glyceraldehyde-phosphate dehydrogenase; ALD: aldolase; TIM: triosephosphate isomerase; MDH: malate dehydrogenase, SOD: superoxide dismutase; FUM: fumarase; $a: \mathrm{n}=5 ; \mathrm{s}_{\mathrm{n}-1}<$ $\pm 10 \% ; b$ : not detectable 

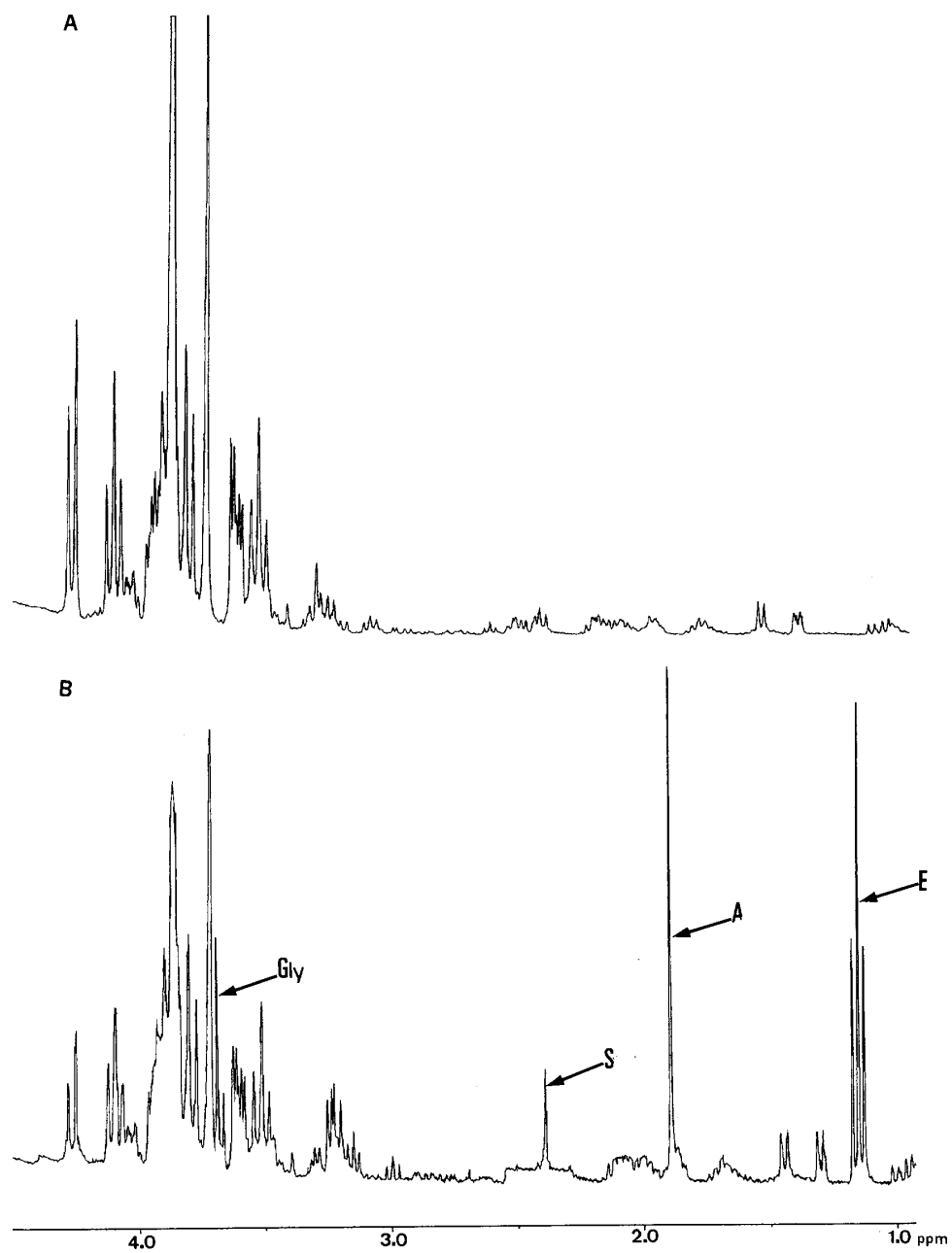

Fig. 2: ${ }^{1} \mathrm{H}$ NMR spectra of flagellates from Amaranthus retroflexus culture. A: fresh culture medium before inoculation of cells; B: spectra for the cell-free culture media, after four days of culture. Metabolites excreted were: E, ethanol (1.12 ppm), A, acetate (1.85 ppm), S, succinate (2.41 ppm) and Gly, glycine (3.32 ppm).

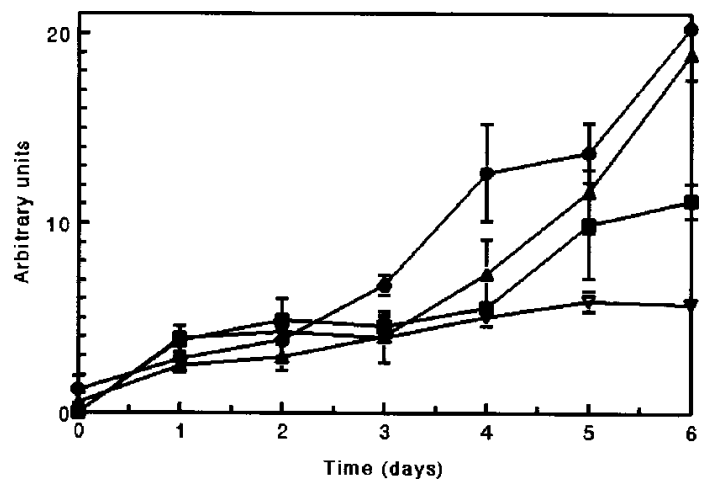

Fig. 3: production and excretion of end metabolites by flagellates isolated from Amaranthus retroflexus during the culture

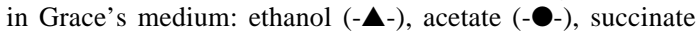
$(-\nabla-)$ and glycine (-口-) detected, in agreement with the non-functionality of the Krebs cycle in these flagellates. After these analyses, determinations were made concerning the association of these enzyme activities with different cell organelles obtained by differential centrifugation of the cell homogenate (Fig. 4). The glycolytic enzymes HK, PFK, PGI, ALD and TIM, together with GK, were associated mainly with the SG. The enzyme involved in $\mathrm{CO}_{2}$ fixation (MDH) was also associated the SG fraction. The enzyme PK was clearly located in the cytosolic fraction (S). As in other flagellates isolated from plants (Fernández-Becerra et al. 1997), the isolate from A. retroflexus presented a clear compartmentalization of the glycolytic pathway, of glycerol metabolism and of $\mathrm{CO}_{2}$ fixation, the enzymes of which 


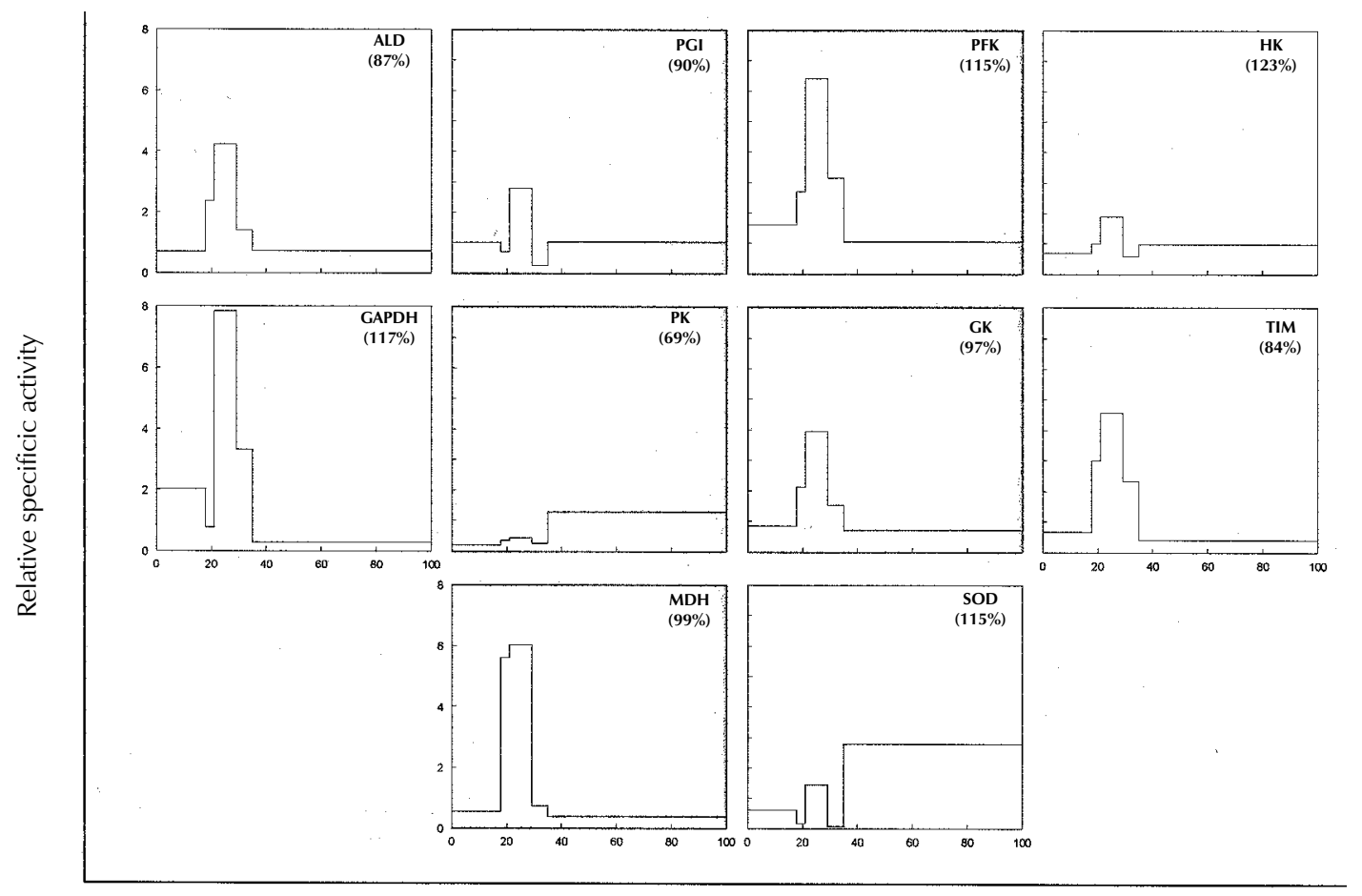

Protein $(\%)$

Fig. 4: distribution profiles of the enzymes from flagellates isolated from Amaranthus retroflexus assayed in the different fractions obtained by differential centrifugation. The fraction are plotted in the order of their isolation, from left to right, nuclear $(\mathrm{N})$, large organelles (LG), small organelles (SG), microsomal (M) and final supernatant (S); ALD: aldolase; PGI: phosphoglucose isomerase; PFK: phosphofructokinase; HK: hexokinase; GAPDH: glyceraldehyde-phosphate dehydrogenase; PK: pyruvate kinase; GK: glycerol kinase; TIM: triosephosphate isomerase; MDH: malate dehydrogenase, SOD: superoxide dismutase

were all present in the glycosomal fraction. Most of these enzymes showed activities substantially higher than those determined in other Phytomonas spp. from either tomato, cherimoya or coconut fruits, as well as in the isolates of Euphorbia characias and Euphorbia hyssopifolia (FernándezBecerra et al. 1997), indicating that the isolates of A. retroflexus have a high capacity to metabolize glucose to pyruvate.

In general, trypanosomatids lack catalase, although these protozoa possess other enzyme systems such as SOD. The presence of this enzyme in species belonging to the genus Phytomonas has been reported previously by us (Fernández-Becerra et al. 1996), but in the present work its activity has been quantified and located within the cell. The enzymatic determination was performed following the method of Paoletti and Mocali (1990), very high enzyme activity being detected in homogenates of A. retroflexus (Table). This activity was found to be mainly associated with the cytosolic fraction (S) (Fig. 4).
This activity and its location were confirmed by isoelectric focusing in polyacrylamide gels (pI 3-9). The activity appears as two isoenzymatic forms with different $\mathrm{pI}$, one of the forms having a $\mathrm{pI}$ of 4.8, while the other form presented a $\mathrm{pI}$ of 7.5 (Fig. 5). The SOD activity detected was insensitive to cyanide but sensitive to hydrogen peroxide, and thus is a Fe-SOD (Fig. 6). By polycrylamide-gradient electrophoresis of 8 to $25 \%$, the Mr of these two bands was determined, at approximately 30 and $51 \mathrm{kDa}$, respectively (Fig. 7).

On the basis of our data, we conclude that the flagellates isolated from A. retroflexus, as those previously isolated from tomato, cherimoya, mango fruits and clover stems, all collected from the same geographical area, present strong morphological and metabolic similarities. Nevertheless, the characterization of the A. retroflexus isolates by isoenzyme and kDNA analysis (Sánchez-Moreno et al. 1998) has revealed that these flagellates are sufficiently dissimilar from the others to suggest that the ones analysed here must be a different species. 


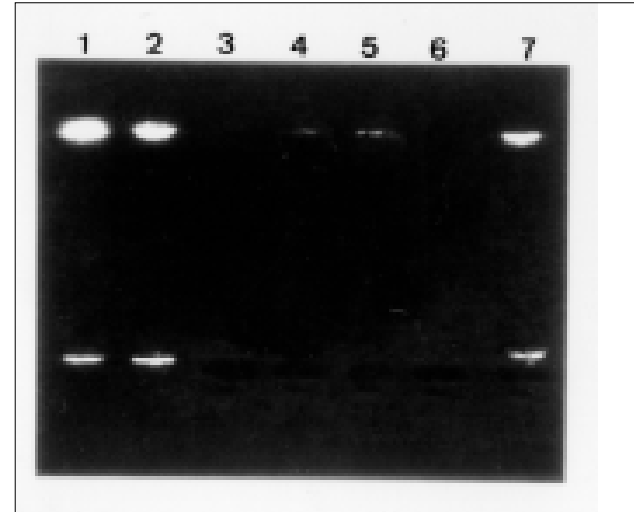

Fig. 5: superoxide dismutase activity in the different fractions obtained from homogenates of Amaranthus retroflexus, on the isoelectrofocus gels (pl. 3-9). lane 1: homogenate, lane 2: post-nuclear fraction, lane 3: postnuclear fraction, lane 4: largeorganelle fraction, lane 5: small-organelle fraction, lane 6: microsomal fraction and lane 7: cytosolic fraction

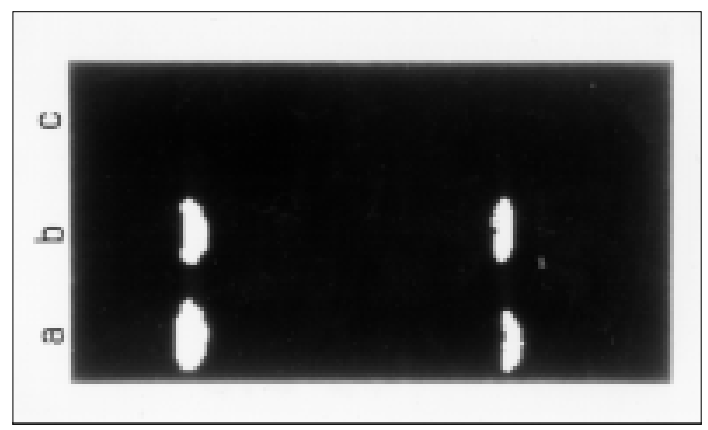

Fig. 6: isoenzymatic profile in IEF 3-9 of the superoxide dismutase activity in the cytosol fraction obtained from Amaranthus retroflexus. a: cytosol fraction; b: cytosol fraction incubated with $5 \mathrm{mM} \mathrm{KCN}$; c: cytosol fraction incubated for $45 \mathrm{~min}$ with $\mathrm{H}_{2} \mathrm{O}_{2} 5 \mathrm{mM}, 0,1 \mathrm{mM}$ disodium EDTA.

\section{REFERENCES}

Bergmeyer HU 1988. Methods of Enzymatic Analysis, Verlag-Chemie/Academic Press, New York, 421 pp.

Beyer WF, Fridovich I 1987. Assaying of SOD activity: some large consequences of minor changes in conditions. Anal Biochem 161: 559-566.

Blum JJ 1993. Intermediary metabolism of Leishmania. Parasitol Today 9: 118-122.

Bradford MM 1976. A rapid and sensitive method for the quantitation of microgram quantities of protein using the principles of protein-dye binding. Anal Biochem 72: 248-254.

Camargo EP 1999. Phytomonas and other trypanosomatid parasites of plants and fruit. Adv Parasitol 42: 29-112.

Donovan C 1909. Kala azar in Madras, especially with regard to its connexion with the dog and the bug (Cnorhinus). Lancet 177: 1495-1496.

Fairlamb AH, Opperdoes F 1986. Carbohydrate metabolism in African trypanosomes with special reference

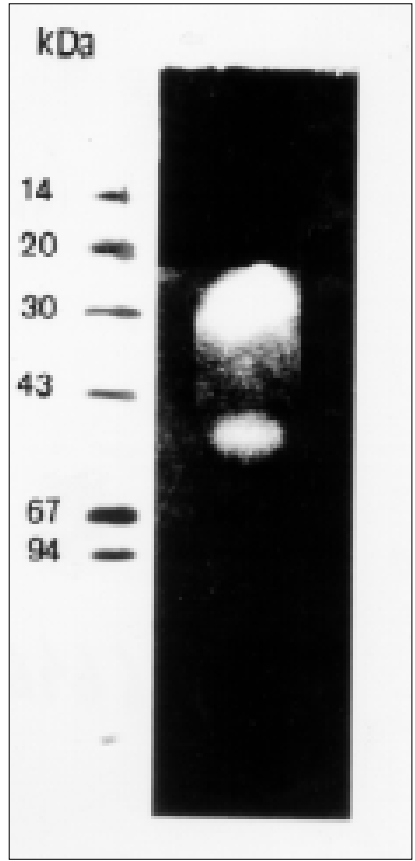

Fig. 7: isoenzymatic profile by polyacrylamide-gradient electrophoresis of 8 to $25 \%$ of the superoxide dismutase activity in the cytosol fraction obtained from Amaranthus retroflexus.

to the glycosome. In MJ Morgan, Carbohydrate Metabolism in Cultured Cells, Plenum, New York/ London, p. 183-224.

Fernández-Becerra C, Osuna A, Muller E, Dollet M, Sánchez-Moreno M 1996. Characterization of isolated from fruits by electrophoretic isoenzymes and kinetoplast DNA analysis. FEMS Microbiol Letters 145: 463-468.

Fernández-Becerra C, Sánchez-Moreno M, Osuna A, Opperdoes F 1997. Comparative aspects of energy metabolism in plant trypanosomatids. J Euk Microbiol 44: 523-529.

Gilroy FV, Edwards MR, Nortom RS, O'Sullivan WJ 1988. Metabolic studies of the protozoan parasite, Crithidia lucidae, using proton nuclear magnetic resonance spectroscopy. Mol Biochem Parasitol 31: 107-116.

Hellemond JJ, Opperdoes F, Tielens GM 1998. Trypanosomatidae produce acetate via a mitochondrial acetate CoA transferase. Proc Natl Acad Sci USA 95: 3036-3041.

Klein RA, Linstead DJ, Wheeler MW 1975. Carbon dioxide fixation in trypanosomatids. Parasitology 71 : 93-107.

Misset O, Bos OJM, Opperdoes F 1986. Glycolytic enzyme of Trypanosoma brucei. Simultaneous purification, intraglycosomal concentrations and physical properties. Eur J Biochem 157: 441-453.

Opperdoes FR, Markos A, Steiger RF 1981. Localization of malate dehydrogenase, adenylate kinase and glycolytic enzymes in glycosomes and the threonine 
pathway in the mitochondrion of cultured procyclics of Trypanosoma brucei. Mol Biochem Parasitol 4: 291-309.

Paoletti F, Mocali A 1990. Determination of superoxide dismutase activity by purely chemical system based on NAD(P)H oxidation. Meth Enzymol 186: 209265.

Sánchez-Moreno M, Lasztity D, Coppens Y, Opperdoes F 1992. Characterization of carbohydrate metabolism and demonstration of glycosomes in a Phytomonas spp. isolated from Euphorbia characias. Mol Biochem Parasitol 54: 185-200.

Sánchez-Moreno M, Fernández-Becerra C, Mascaró C, Rosales MJ, Dollet M, Osuna A 1995. Isolation, in vitro culture, ultrastructure study, and characterization by lectin-agglutination test of Phytomonas isolated from tomatoes (Lycopersicum esculentum) and cherimoyas (Annona cherimolia) in southeastern
Spain. Parasitol Res 81: 575-581.

Sánchez-Moreno M, Fernández-Becerra C, FernándezRamos C, Luque F, Rodriguez-Cabezas MN, Dollet M, Osuna A 1998. Trypanosomatid protozoa in plants of southeastern Spain: characterization by analysis of isoenzymes, kinetoplast DNA, and metabolic behaviour. Parasitol Res 84: 354-361.

Steiger RF, Opperdoes FR, Bontemps J 1980. Subcellular fractionation of Trypanosoma brucei bloodstream forms with special reference to hydrolases. Eur J Biochem 105: 163-175.

Van Schaftingen E, Opperdoes FR, Hers HG 1985. Stimulation of Trypanosoma brucei piruvate kinase by fructose 2,6-bisphosphate. Eur J Biochem 153: 403-406.

Uttaro AD, Sánchez-Moreno M, Opperdoes F 1997. Genus-specific biochemical markers for Phytomonas spp. Mol Biochem Parasitol 90: 337-342. 
